\title{
Automated indexation of metabolic changes in Alzheimer's mice using a voxel-wise approach combined to an MRI-based 3D digital atlas
}

\author{
Jessica Lebenberg, Anne-Sophie Hérard, Albertine Dubois, \\ Marc Dhenain, Philippe Hantraye and Thierry Delzescaux
}

\begin{abstract}
Brain glucose uptake was examined in transgenic mice relevant to Alzheimer's disease (APP/PS1) and their control littermates (PS1). Glucose distribution in the brain of the resting animals was measured using 3D-reconstructed autoradiography and analysed by a voxel-wise approach using SPMMouse combined to an MRI-based 3D digital atlas. Prompt and direct indexation of metabolic changes between the two groups was achieved, showing both hypo- and hypermetabolism of glucose in the brain of APP/PS1 mice. We confirm and extend previous study, since we identified brain structures affected in this pathological model and demonstrate glucose uptake changes in structures like the olfactory bulb. Our results pave the way to complete and accurate examination of functional data from cerebral structures involved in models of neurodegenerative diseases.
\end{abstract}

\section{INTRODUCTION}

Murine models are commonly used to improve our understanding of the pathophysiology of human diseases and to determine the effects of drugs. In the field of neurodegenerative diseases like Alzheimer's Disease, brain images are analyzed to evaluate the anatomofunctional changes involved during the evolution of the pathology. To accurately study brain function, ex vivo autoradiography remains the gold standard technique [1]. Brain metabolic changes can be determined by analysing these autoradiographs using a voxel-wise approach. This permits statistical comparisons between groups involved in the study at the single-voxel scale and provides "clusters of voxels" representing the functional changes for a given statistical significant level. This can be achieved by using dedicated tools such as the Statistical Parametric Mapping (SPM) software (Wellcome Department of Imaging Neuroscience, London, UK). Initially developed for clinical studies, few functional studies on rodent models have been performed with SPM yet [2], [3]. A recent adaptation of this tool has been dedicated to small animal imaging, SPMMouse, and used in a morphometrical study of transgenic mice [4]. The localization of clusters refers to bregma coordinates based on anatomical atlases [5]. Too rough to give an accurate localization [4], expert visual identification is required. This operator-dependant and time-consuming task quickly becomes tedious if many small clusters are detected and can lead to misinterpretations. To overcome these limitations, we propose to automatically

J. Lebenberg, A.S. Hérard, M. Dhenain, P. Hantraye and T. Delzescaux are with the Medical Image Research Center (MIRCen), URA CEA-CNRS 2210, CEA-DSV-I2BM, 18 route du Panorama, F-92265 Fontenay Aux Roses Cedex, France thierry. delzescaux@cea.fr

A. Dubois is with the Service Hospitalier Frédéric Joliot (SHFJ), INSERM U803, CEA-DSV-I2BM, F-91401 Orsay Cedex, France index clusters using digital atlas-based segmentations previously registered to the study-specific template created for the voxel-wise analysis. Based on a method described in [6], the reliability of our approach was assessed qualitatively and quantitatively by comparing atlas-based with manual segmentations. The method was developed in a preliminary study on $\mathrm{APP}_{S L} / \mathrm{PS} 1_{M 146 L}$ (Alzheimer's disease model) and PS1 $1_{M 146 L}$ (control littermates) transgenic mice. Functional parameters determined using this new approach were compared with results previously described in the literature.

\section{MATERIALS}

\section{A. Animals and 3D-reconstruction of brain data}

Our method was applied to $4 \mathrm{APP}_{S L} / \mathrm{PS} 1_{M 146 L}(64 \pm 1$ week-old) and 3 PS $1_{M 146 L}$ mice $(65 \pm 2$ week-old), with a $\mathrm{C} 57 \mathrm{Bl} / 6$ genetic background [7]. All the procedures were carried out in accordance with the recommendations of the EEC (directive 86/609/EEC) and the French National Committee (decree 87/848) for the use of laboratory animals. $\left[{ }^{14} \mathrm{C}\right]$-2-deoxyglucose (2DG) uptake was measured in the brain of resting mice using quantitative autoradiography. Right hemispheres (without outer olfactory bulb and cerebellum) were cut into $20-\mu \mathrm{m}$ serial coronal sections for ex vivo analysis. Every fourth serial section was mounted on a glass slide and exposed to autoradiographic film. The same sections were then processed for Nissl staining to obtain anatomical information. Images from the brain surface (block-face), corresponding to the processed sections, were recorded before sectioning using a digital camera with an in-plane resolution of $27 \times 27 \mu \mathrm{m}^{2}$.

Autoradiographs and histological sections were digitized using a flatbed scanner (1200 dpi in-plane resolution, pixel size $21 \times 21 \mu \mathrm{m}^{2}$ ). Using BrainRAT (toolbox of the free software BrainVISA, http://brainvisa.info/) and the method presented in [3], three spatially coherent 3Dreconstructed post mortem volumes (block-face, autoradiographic and histological volumes) were obtained for each mouse in a common frame of reference. Additional details can be found in [3].

\section{B. MRI-based 3D digital mouse brain atlas}

We used the MRI-based 3D digital atlas of the Center for In Vivo Microscopy ( http://www. civm. duhs.duke. edu/), which was derived from $\mathrm{T} 1$ and $\mathrm{T} 2$-weighted 3D MRI (9.4 T) of 6 young adult (9-12 weeks) C57B1/6J mice. MRI were recorded within the skull after active staining of the brain [8]. The isotropic scan resolutions were $21.5 \mu \mathrm{m}$ 
and $43 \mu \mathrm{m}$ for $\mathrm{T} 1$ and $\mathrm{T} 2$ images respectively. Thirty-three anatomical regions of interest (ROI) were segmented [9]. For this study, we performed a manual segmentation using the atlas-based segmentation of the cortex, to add the cingulate and retrospenial cortex to the ROI list.

\section{Methods}

A. Detection of metabolic changes using a voxel-wise approach

The detection of metabolic changes in mouse brains was realized using SPMMouse software [4]. To analyze the 3Dreconstructed autoradiographic data, a study-specific template based on block-face volumes was first created [Fig. 1a)] as described in [3]. Autoradiographic volumes were then spatially normalized to the study-specific template using the same software. The background and ventricles were subtracted from the analysis. Using a two-sample t-test, two contrasts were evaluated separately to produce statistical parametric maps from the gray level intensities of autoradiographic volumes: 1) voxels with lower 2DG uptake regions in APP/PS1 than in PS1 mice (APP/PS1 hypometabolism); 2) voxels with higher $2 \mathrm{DG}$ uptake regions in APP/PS1 than in PS1 mice (APP/PS1 hypermetabolism) [Fig. 1b)]. As in [3], the clusters of voxels representing metabolic changes were detected considering $\mathrm{P}<0.01$ (uncorrected for multiple comparisons) as statistical significance level and containing at least 1500 contiguous voxels (cluster size $\gtrsim 0.05 \mathrm{~mm}^{3}$ ).

\section{B. Automated indexation of clusters using the digital atlas}

Digital atlas-based segmentations were used to automatically localize the clusters. Using the method described in [6], we registered the T1-weighted MRI, associated to the digital atlas, to the study-specific template using linear and non-linear transformations. We then warped the digital atlas using the estimated deformation parameters [Fig. 1c)].

Registration accuracy was qualitatively assessed by visual inspection of the superimposition of the inner and outer contours of registered MRI (extracted using a Deriche Filter) on the study-specific template. To quantitatively evaluate the data registration, the concordance between atlas-based segmentations and ROIs manually delineated within histological volumes was measured with overlapping criteria. The hippocampus, cortex, striatum (merging of nucleus accumbens and caudate putamen ROIs), corpus callosum and substantia nigra were manually delineated on one APP/PS1 and one PS1 mouse brain histology (Nissl). Block-face volumes corresponding to these histological volumes were registered to the study-specific template using the method described in [6]. The estimated deformation parameters were applied on manual delineations to register them to the studyspecific template. The volume differences, sensitivity and Dice coefficient between registered atlas-based and manual ROIs were computed as concordance indices.

Finally, the registered atlas was superimposed to the statistical parametric maps. The clusters representing metabolic changes were thus automatically classified within atlas-based ROIs [Fig. 1d)]. We also computed the percentage of cluster voxels overlapping with ROI voxels as it was done in humans [10].

\section{Automated functional analysis}

Each cluster detected by the voxel-wise approach and indexed by the atlas [Fig. 1d)] allowed measurement of averaged 2DG uptake with associated standard error mean $(\mathrm{nCi} / \mathrm{g}$, mean $\pm \mathrm{SEM})$ on the autoradiographic volumes of all APP/PS1 and PS1 mice. Metabolic differences (in \%) between strains were calculated from these values for each brain structure and a two-sample unpaired t-test was performed (significant level at $5 \%$ ).

\section{RESUlTS}

\section{A. Digital atlas and study-specific template registration}

Visual inspection attested a good registration of the inner structures (as the hippocampus and corpus callosum) and

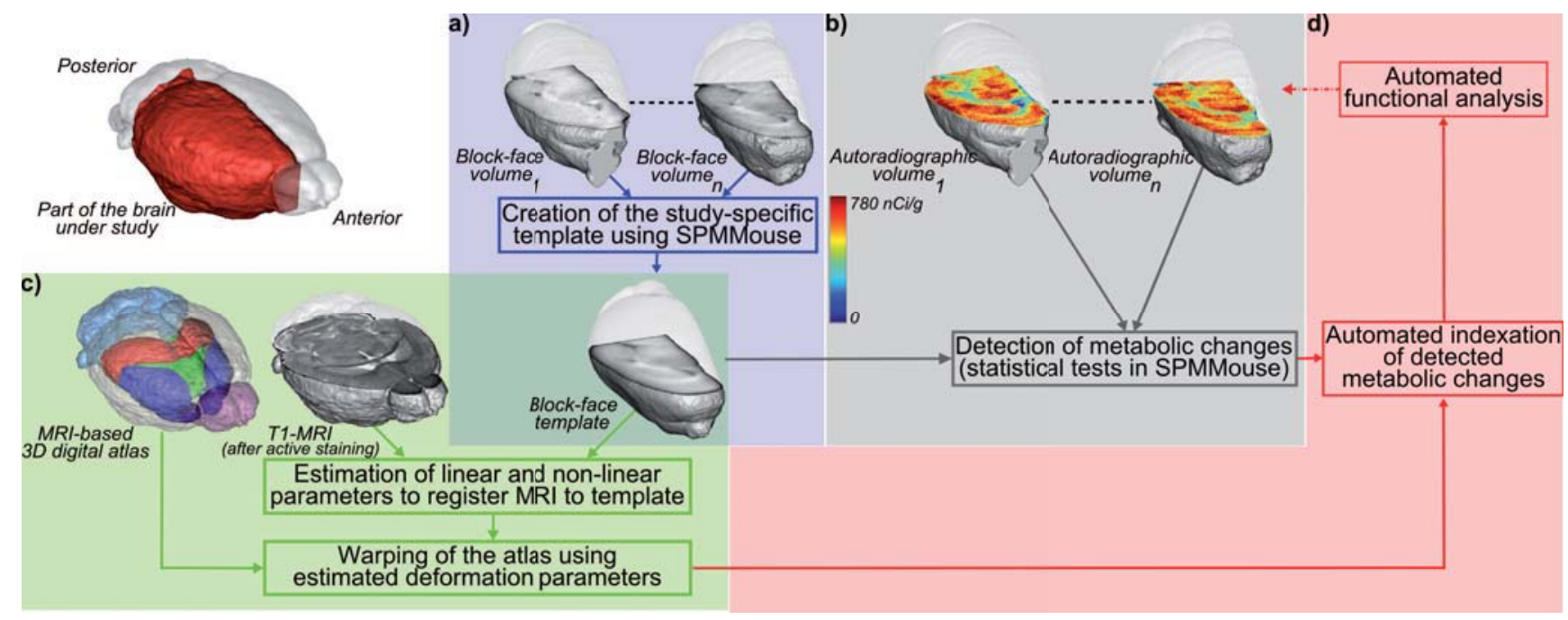

Fig. 1. Method to automatically index and analyze metabolic changes detected by a voxel-wise approach using an MRI-based digital atlas 
external contours of MRI to the study-specific template (not shown). Table I displays volumes differences, Dice coefficients and sensitivity computed for the cortex, corpus callosum, hippocampus, striatum and substantia nigra of one PS1 and one APP/PS1 mouse registered to the study-specific template. This table presents similar overlapping scores as in the study described in [6] where the same atlas was used to analyze post mortem volumes non registered to a template. This digital atlas can thus analyze the study-specific template as well as the original post mortem volumes.

TABLE I

Computation of Volume differences $\left(\Delta_{V}\right)$, Dice Coefficient $(\kappa)$ And Sensitivity (SE) to EVAluate the CONCORDANCE BETWEen REGISTERED ATLAS-BASED AND MANUALLY DELINEATED ROIS

\begin{tabular}{l|ccc|cccc}
\hline & \multicolumn{3}{c|}{$\begin{array}{c}\text { PS1 mouse } \\
\text { (ctrl) }\end{array}$} & \multicolumn{3}{c}{$\begin{array}{c}\text { APP/PS1 mouse } \\
\text { (AD model) }\end{array}$} \\
\hline \hline & $\Delta_{V}$ & $\kappa$ & $\mathrm{Se}$ & $\Delta_{V}$ & $\kappa$ & $\mathrm{Se}$ \\
Cortex & 0.07 & 0.83 & 0.80 & 0.20 & 0.82 & 0.74 \\
Corpus callosum & 0.25 & 0.55 & 0.49 & 0.07 & 0.31 & 0.32 \\
Hippocampus & 0.02 & 0.82 & 0.81 & 0.15 & 0.77 & 0.83 \\
Striatum & 0.01 & 0.82 & 0.82 & 0.08 & 0.77 & 0.80 \\
Substantia nigra & 0.18 & 0.51 & 0.47 & 0.03 & 0.58 & 0.57 \\
\hline
\end{tabular}

\section{B. Clusters displaying metabolic changes}

Nine and eight clusters showing respectively hypo- and hypermetabolism in APP/PS1 mice were detected using statistical tests designed in SPMMouse. Figs. 2a) and b) display these lower and higher 2DG uptake regions in APP/PS1 than PS1 mice respectively and show these metabolic changes are distributed throughout the hemisphere.

\section{Automated indexation of detected clusters}

Table II presents the percentage of cluster voxels overlapping with atlas-ROI voxels. The clusters 3,8 and 9 and the cluster 1 representing respectively hypo- and hypermetabolism in APP/PS1 mice were totally included in the "rest of cortex".

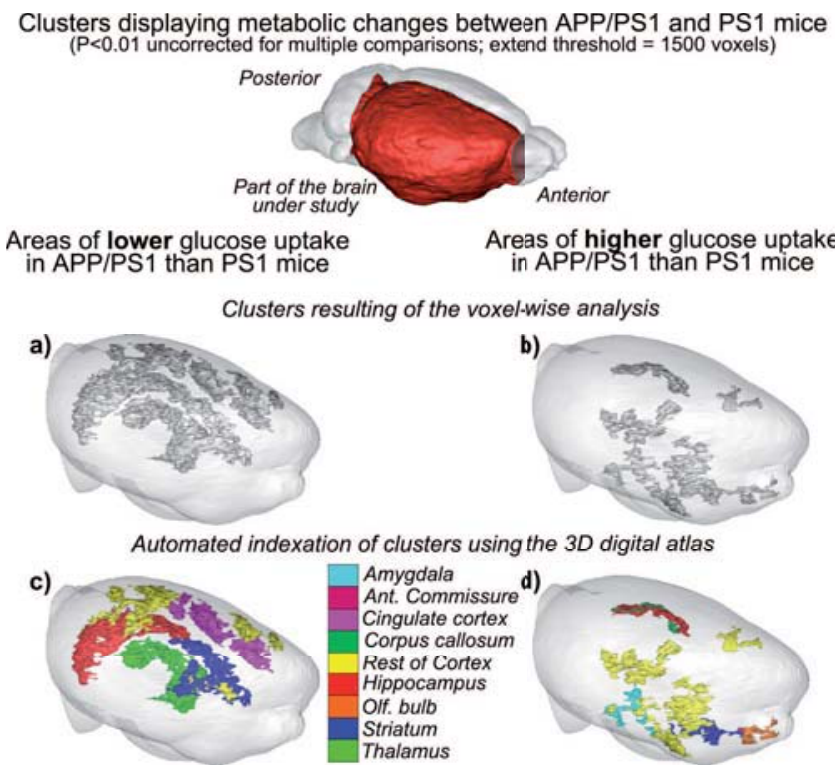

Fig. 2. 3D representation of clusters showing metabolic changes before $(\mathrm{a}$ and $\mathrm{b})$ and after $(\mathrm{c}$ and $\mathrm{d})$ the automated indexation
Expert visual inspection of superimposition of clusters onto atlas and of clusters onto histological volumes led to conclude to few misregistrations of the atlas to the template. They can be illustrated by the following examples. In the statistical parametric map representing APP/PS1 hypometabolism, the clusters 2 and 7 and the cluster 6 should not overlap the rest of cortex and the thalamus respectively. In the statistical parametric map representing APP/PS1 hypermetabolism, the cluster 2 should not overlap the amygdala and the corpus callosum, the clusters 4 and 5 and the clusters 6 and 8 should not overlap the rest of cortex and the striatum respectively. Clusters belonging to the ventricles (cluster 7, APP/PS1 hypometabolism) were also considered as misregistered (see paragraph III-A). The concerned part of cluster 7 was merged with the closest ROI, i.e. the striatum for the functional analysis. Visual inspection confirmed a reliable spreading over several ROIs of all other clusters, which were automatically splitted into subclusters.

According to these results, the clusters and subclusters displaying hypo- and hypermetabolism in APP/PS1 mice were automatically indexed using the atlas-based segmentations. A 3D representation of the indexed clusters is displayed in Figs. 2c) and d).

\section{Automated functional analysis}

Table III presents for each strain mean 2DG uptake $(\mathrm{nCi} / \mathrm{g})$ measured in all indexed clusters and subclusters and percentage difference computed between APP/PS1 and PS1 mice. Table III A) shows that average activities measured within clusters and subclusters representing the hypometabolism were $\sim 18 \%$ lower in APP/PS1 than PS1 mice. Within subclusters representing the hypermetabolism

TABLE II

PERCENTAGE OF CLUSTER VOXELS OVERLAPPING WITH ATLAS-ROI VOXELS $(\mathrm{P}<0.01$ UNCORRECTED FOR MULTIPLE COMPARISONS; EXTEND THRESHOLD $=1500$ VOXELS

A)Clusters showing lower glucose uptake areas in APP/PS1 than PS1 mice

\begin{tabular}{|c|c|c|c|c|c|c|c|c|c|}
\hline $\begin{array}{l}\text { Atlas Clusters } \\
\text { ROI }\end{array}$ & 1 & 2 & 3 & 4 & 5 & 6 & 7 & 8 & 9 \\
\hline Cing./Retro. cortex & - & 98.5 & - & 72.2 & - & 2.9 & - & - & - \\
\hline Rest of Cortex & 9.2 & 0.1 & 100 & 27.7 & - & 10.0 & 0.2 & 100 & 100 \\
\hline Hippocampus & - & - & - & - & - & 84.4 & - & - & - \\
\hline Striatum & 89.4 & - & - & - & - & - & 51.0 & - & - \\
\hline Thalamus & - & - & - & - & 99.9 & 0.1 & - & - & - \\
\hline Ventricles & - & - & - & - & - & - & 44.7 & - & - \\
\hline Rest of brain & 1.4 & 1.4 & - & 0.1 & 0.1 & 2.7 & 4.1 & - & - \\
\hline
\end{tabular}

B)Clusters showing higher glucose uptake areas in APP/PS1 than PS1 mice

\begin{tabular}{l|cccccccc} 
Atlas & & & & & & & \\
ROI & 1 & 2 & 3 & 4 & 5 & 6 & 7 & 8 \\
Amygdala & - & 0.2 & 5.1 & - & - & - & - & 73.4 \\
Ant. Commissure & - & - & - & 5.4 & - & - & - & - \\
Corpus callosum & - & 0.7 & - & - & 25.2 & 20.3 & - & - \\
Rest of Cortex & 100 & 99.1 & 91.5 & 1.9 & 0.4 & 79.1 & 77.1 & 26.5 \\
Hippocampus & - & - & - & - & 74.4 & - & - & - \\
Olf. bulb & - & - & - & 45.4 & - & - & - & - \\
Striatum & - & - & 3.3 & 47.3 & - & 0.7 & 22.9 & 0.1 \\
\hline
\end{tabular}


TABLE III

IDENTIFICATION (ATLAS ROI AND CLUSTER) AND QUANTIFICATION OF METABOLIC CHANGES ( $\overline{\text { MEAN }} \pm$ STANDARD ERROR MEAN IN NCI/G) AND PERCENTAGE DIFFERENCE BETWEEN APP/PS1 AND PS1 MICE

\begin{tabular}{lcccc}
\hline $\begin{array}{l}\text { Atlas } \\
\text { ROI }\end{array}$ & Cluster & $\begin{array}{c}\text { PS1 mice } \\
(\mathbf{n}=\mathbf{3})\end{array}$ & $\begin{array}{c}\text { APP/PS1 mice } \\
(\mathbf{n}=\mathbf{4})\end{array}$ \\
\hline \hline A) Lower glucose & uptake areas in & APP/PS1 than in PS1 mice \\
Cing./Retro. & 2 & $366.96 \pm 13.98$ & $276.34 \pm 3.02-24.69$ \\
cortex & 4 & $318.87 \pm 4.32$ & $261.92 \pm 1.14-17.86$ \\
& 6 & $311.03 \pm 7.80$ & $247.50 \pm 5.13-20.43$ \\
Rest of & 1 & $290.08 \pm 2.37$ & $247.67 \pm 2.66-14.62$ \\
Cortex & 3 & $319.97 \pm 3.86$ & $270.24 \pm 3.49-15.54$ \\
& 4 & $326.16 \pm 3.02$ & $274.41 \pm 1.93-15.87$ \\
& 6 & $310.38 \pm 3.94$ & $254.50 \pm 3.48-18.00$ \\
& 8 & $336.98 \pm 6.09$ & $275.56 \pm 2.24-18.23$ \\
Hippocampus & 6 & $338.87 \pm 3.66$ & $281.03 \pm 5.85-17.07$ \\
Striatum & 1 & $309.26 \pm 3.21$ & $239.33 \pm 3.19-20.60$ \\
& 7 & $285.10 \pm 2.64$ & $256.03 \pm 0.48-17.21$ \\
Thalamus & 5 & $333.27 \pm 9.43$ & $264.96 \pm 1.75-20.50$ \\
\hline \hline B) Higher glucose & uptake areas in & APP/PS1 than in PS1 mice \\
Amygdala & 3 & $241.75 \pm 4.42$ & $288.85 \pm 7.16+19.48$ \\
& 8 & $206.68 \pm 2.34$ & $243.94 \pm 2.01+18.03$ \\
Ant.Comm. & 4 & $221.52 \pm 2.45$ & $278.05 \pm 1.31+25.52$ \\
Corpus & 5 & $180.00 \pm 4.21$ & $229.67 \pm 2.05+27.60$ \\
callosum & 6 & $241.84 \pm 0.70$ & $282.86 \pm 3.60+16.96$ \\
Rest of & 1 & $171.28 \pm 14.48$ & $278.26 \pm 6.22+62.46$ \\
Cortex & 2 & $240.66 \pm 2.35$ & $284.31 \pm 2.18+18.14$ \\
& 3 & $256.97 \pm 0.52$ & $309.95 \pm 4.52+20.62$ \\
& 6 & $265.62 \pm 2.16$ & $301.03 \pm 1.45+13.33$ \\
& 7 & $271.06 \pm 7.63$ & $344.79 \pm 7.32+27.20$ \\
Hippocampus & 5 & $218.69 \pm 2.02$ & $257.79 \pm 1.49+17.88$ \\
Olf. Bulb & 4 & $217.98 \pm 3.94$ & $241.54 \pm 2.43+24.16$ \\
Striatum & 3 & $252.90 \pm 5.18$ & $281.99 \pm 3.17+29.37$ \\
& 4 & $258.01 \pm 0.73$ & $307.11 \pm 1.94+19.03$ \\
& 7 & $275.53 \pm 5.63$ & $349.46 \pm 6.37+26.83$ \\
\hline & & &
\end{tabular}

[Table III B)], the average activities were $~ 24 \%$ higher in APP/PS1 than PS1 mice. The t-test computed to compare the two strains revealed statistically significant functional differences at the level of clusters $(\mathrm{P} \leq 0.05)$.

\section{Discussion AND CONCLUSion}

Metabolic changes in the brain of transgenic mice relevant to Alzheimer's disease were detected by voxel-wise analysis using SPMMouse. The use of a mouse brain digital atlas registered to the study-specific template created for the voxelwise analysis reduced the time required for indexing the position of clusters in the brain, as well as the risk of misinterpretations in their localization.

To register the digital atlas to the statistical maps the method described in [6] was applied using a study-specific template as reference image for the registration process. The overlapping criteria computed between atlas-based and manual segmentation registered to the study-specific template attested the reliability of the atlas and authorized its use [6]. A careful analysis of thin and deep structures is required since the overlapping scores obtained for the corpus callosum and substantia nigra are low.

Indexation and distribution in the mouse brain of metabolic changes between strains were promptly provided using the superimposition of the atlas to the statistical maps and the computation of the percentage of cluster voxels overlapping with atlas-ROI voxels. Our approach permits an automated split of clusters spread over several ROIs into subclusters using the atlas-based segmentations, a task difficult to achieve manually. Additional tests will be realized by changing the statistical settings to evaluate the impact on cluster size and localization.

The hypermetabolism in APP/PS1 mice detected in white matter could be due to an atlas misregistration as suggested by overlapping criteria. Nevertheless, this can also reflect the corpus callosum volume reduction [1] or a partial volume effect [11]. The hypermetabolism in the olfactory bulb of APP/PS1 mice shows that this structure was not entirely removed and suggests compensatory mechanisms [12]. This also underlines that our approach permits to reveal structures potentially involved in Alzheimer's disease that would not have been noticed with a visual identification of clusters [3].

Finally, registration of the digital atlas to the study-specific template created for a voxel-wise analysis could be used to limit statistical tests to selected voxels and then allow the correction of these tests and the refinement of results [13].

\section{ACKNOWLEDGMENTS}

We thank the Sanofi-Aventis Neurodegenerative Disease Group for providing the transgenic mice.

\section{REFERENCES}

[1] J. Valla, L. Schneider, and E. M. Reiman, "Age- and transgene-related changes in regional cerebral metabolism in PSAPP mice.," Brain Res, vol. 1116, pp. 194-200, Oct 2006.

[2] P. Nguyen et al. "Statistical parametric mapping applied to an autoradiographic study of cerebral activation during treadmill walking in rats," NeuroImage, vol. 23, no. 1, pp. 252-9, 2004.

[3] A. Dubois et al. "Detection of significant changes in regional cerebral glucose uptake in APP/PS1 mouse model of Alzheimer's disease using voxel-wise statistical analysis," Neuroimage, vol. 51, pp. 586-598, Jun 2010.

[4] S. J. Sawiak et al. "Voxel-based morphometry in the R6/2 transgenic mouse reveals differences between genotypes not seen with manual 2D morphometry.," Neurobiol Dis, vol. 33, pp. 20-27, Jan 2009.

[5] G. Paxinos and K. Franklin, The Mouse Brain inStereotaxic Coordinates. 2004.

[6] J. Lebenberg et al. "Validation of MRI-based 3D digital atlas registration with histological and autoradiographic volumes: An anatomofunctional transgenic mouse brain imaging study.," Neuroimage, vol. 51, pp. 1037-1046, Jul 2010.

[7] B. Delatour, M. Guégan, A. Volk, and M. Dhenain, "In vivo MRI and histological evaluation of brain atrophy in APP/PS1 transgenic mice.," Neurobiol Aging, vol. 27, pp. 835-847, Jun 2006.

[8] G. A. Johnson et al. "High-throughput morphologic phenotyping of the mouse brain with magnetic resonance histology." Neuroimage, vol. 37, pp. 82-89, Aug 2007.

[9] A. A. Sharief, A. Badea, A. M. Dale, and G. A. Johnson, "Automated segmentation of the actively stained mouse brain using multi-spectral MR microscopy.," Neuroimage, vol. 39, pp. 136-145, Jan 2008.

[10] N. Tzourio-Mazoyer et al. "Automated anatomical labeling of activations in SPM using a macroscopic anatomical parcellation of the MNI MRI single-subject brain.," Neuroimage, vol. 15, pp. 273-289, Jan 2002.

[11] J. Valla et al. "Effects of image resolution on autoradiographic measurements of posterior cingulate activity in pdapp mice: implications for functional brain imaging studies of transgenic mouse models of alzheimer's disease.," Neuroimage, vol. 16, pp. 1-6, May 2002.

[12] D. W. Wesson, E. Levy, R. A. Nixon, and D. A. Wilson, "Olfactory dysfunction correlates with amyloid-beta burden in an alzheimer's disease mouse model.," J Neurosci, vol. 30, pp. 505-514, Jan 2010.

[13] C. R. Genovese, N. A. Lazar, and T. Nichols, "Thresholding of statistical maps in functional neuroimaging using the false discovery rate.," Neuroimage, vol. 15, pp. 870-878, Apr 2002. 